\title{
Bone marrow mesenchymal stem cell-derived extracellular vesicles improve the survival of transplanted fat grafts
}

\author{
HE HUANG ${ }^{*}$, SHAOQING FENG ${ }^{*}$, WENJIE ZHANG, WEI LI, PENG XU, XIANGSHENG WANG and AI AI \\ Department of Plastic and Reconstructive Surgery, Shanghai 9th People's Hospital, Shanghai Jiao Tong University School \\ of Medicine, Shanghai 200011, P.R. China
}

Received May 23, 2016; Accepted April 4, 2017

DOI: $10.3892 / \mathrm{mmr} .2017 .6972$

\begin{abstract}
Autologous fat grafting is a promising surgical technique for soft tissue augmentation, reconstruction and rejuvenation. However, it is limited by the low survival rate of the transplanted fat, due to the slow revascularization of such grafts. Previous studies have demonstrated that bone marrow mesenchymal stem cell-derived extracellular vesicles (BMSC-EVs) are proangiogenic. The present study aimed to investigate whether BMSC-EVs could improve the survival of transplanted fat grafts. Extracellular vesicles were isolated from the supernatant of cultured rat bone marrow mesenchymal stem cells, and characterized by flow cytometry and scanning electron microscopy. Their proangiogenic potential was measured in vitro using tube formation and cell migration assays. Subsequently, human fat tissue grafts, alongside various concentrations of BMSC-EVs, were subcutaneously injected into nude mice. A total of 12 weeks following transplantation, the mice were sacrificed and the grafts were harvested. The grafts from the experimental group had a higher survival rate and an increased number of vessels compared with grafts from the control group, as demonstrated by tissue volume, weight and histological analyses. Reverse transcription-quantitative polymerase chain reaction analysis indicated that the expression levels of proangiogenic factors were increased in the experimental group compared with in the control group, thus suggesting that BMSC-EVs may promote neovascularization by stimulating the secretion of proangiogenic factors. The present study is the first, to the best
\end{abstract}

Correspondence to: Dr Wenjie Zhang or Dr Wei Li, Department of Plastic and Reconstructive Surgery, Shanghai 9th People's Hospital, Shanghai Jiao Tong University School of Medicine, 639 Zhizaoju Road, Shanghai 200011, P.R. China

E-mail: zhangwenjie051116@163.com

E-mail: liwei051216@sina.com

*Contributed equally

Key words: BMCs, autologous grafting, extracellular vesicles, neovascularization, proangiogenic factors of our knowledge, to demonstrate that supplementation of fat grafts with BMSC-EVs improves the long-term retention and quality of transplanted fat.

\section{Introduction}

Autologous fat grafting is a promising surgical technique for soft tissue augmentation, reconstruction and rejuvenation. Fat tissue is abundant, readily available and relatively low cost, and can be harvested with minimal trauma to the donor site. Fat grafts have been extensively used in numerous fields, including the treatment of Romberg's disease, depressed scars, wrinkles, senile atrophy, pitting acne, breast augmentation, and hand and face rejuvenation (1). Despite these uses, transplanted fat often has a low survival rate, and adipose tissue can be quickly resorbed and replaced with fibrous tissue and oil cysts (2-4). Fat graft retention rates have been reported to range between 10 and $90 \%(5,6)$. Several theories have been suggested to explain this phenomenon $(7,8)$. At present, the generally accepted explanation for the decreasing fat volume is insufficient blood supply following transplantation (8-10). Therapeutic approaches that stimulate angiogenesis, including co-transplanting fat with proangiogenic growth factors, endothelial progenitor cells and mesenchymal stem cells (MSCs) from various sources, have significantly improved fat graft retention (11-16). Such co-transplanted cells likely promote neovascularization via the paracrine action of proangiogenic factors, a mechanism that has also been observed during the treatment of other ischemic diseases (17-19).

In addition to cell-based therapies for the treatment of ischemic diseases, interest in the vesicles released by these cultured cells has garnered interest. Extracellular vesicles $(\mathrm{EVs})$, which are released by almost all cells, are normally comprised of three types: Microvesicles, exosomes and apoptotic bodies (20). Furthermore, EVs have been detected in various body fluids, including blood, malignant pleural effusion, urine, saliva and cerebrospinal fluid (21). Previously, EVs were considered inert cellular debris, the consequence of cell damage or the result of dynamic plasma membrane turnover. However, EVs have more recently been reported to serve an important role in intercellular communication (22). The structure of EVs consists of a lipid bilayer membrane, similar to the cellular membrane, which envelops host-specific proteins, mRNAs and microRNAs. Numerous studies have investigated 
the role of EVs in tissue repair and regeneration $(18,22,23)$. In addition, animal studies have demonstrated that the systemic application of EVs derived from cultured bone marrow MSCs (BMSC-EVs) protects cells during acute kidney injury and cardiovascular disease $(18,23,24)$. It has been suggested that BMSC-EVs may protect injured tissues by inducing angiogenesis $(18,24)$.

Based on the proangiogenic potential of BMSC-EVs, the present study hypothesized that co-transplantation of fat with BMSC-EVs during grafting would prevent fat cell death and stimulate neovascularization, thus helping to prevent graft resorption. To test this hypothesis, BMSC-EVs were collected from the supernatant of cultured rat BMSCs using ultra-centrifugation. Subsequently, human fat tissue, combined with various concentrations of BMSC-EVs, was subcutaneously injected into nude mice, and the effects were evaluated 12 weeks post-transplantation.

\section{Materials and methods}

Cell culture and isolation of EVs. A total of 80 male Wistar rats (4 weeks old) weighing 90-110 g were purchased from the Shanghai Chuansha Experimental Animal Raising Farm (Shanghai, China). The animal study protocols were approved by the Animal Care and Experiment Committee of Shanghai Jiao Tong University School of Medicine (Shanghai, China). They were housed under a temperature of $20-26^{\circ} \mathrm{C}$, a relative humidity of $40-70 \%$ and a day/night cycle of $12 / 12 \mathrm{~h}$, with free access to food and water. BMSCs were cultured as previously described (25). Briefly, rat bone marrow cells were extracted from the femurs of male Wistar rats. To remove the majority of the non-adherent blood cells, primary culture of bone marrow cells was performed by seeding cells at $1.6 \times 10^{4}$ cells $/ \mathrm{cm}^{2}$ in Dulbecco's modified Eagle's medium (DMEM; Invitrogen; Thermo Fisher Scientific, Inc., Waltham, MA, USA) supplemented with $10 \%$ fetal bovine serum (FBS; Hyclone; GE Healthcare Life Science, Logan, UT, USA) and $0.2 \%$ penicillin/streptomycin (Sigma-Aldrich; Merck KGaA, Darmstadt, Germany) at $37^{\circ} \mathrm{C}$. The medium was changed every 3 days. After 6-7 days of culture the primary adherent cells (P0) were harvested using trypsin/EDTA $(0.25 \% \mathrm{w} / \mathrm{v}$ trypsin and $0.02 \%$ EDTA; Invitrogen; Thermo Fisher Scientific, Inc.), and were subcultured at $1.6 \times 10^{4}$ cells $/ \mathrm{cm}^{2}$ in a $10-\mathrm{cm}$ diameter tissue culture dish in $10 \mathrm{ml}$ DMEM containing $10 \% \mathrm{FBS}$. The cells were passaged in the same manner every 3 days. Passage 4 cells were evaluated for typical MSC characteristics, including the cell surface marker expression profile and multi-lineage differentiation potential (data not shown), and were used for the collection of EVs. Once the cells reached $70 \%$ confluence, they were cultured in serum-free media under hypoxic conditions $\left(94 \% \mathrm{~N}_{2}, 5 \% \mathrm{CO}_{2}\right.$ and $\left.1 \% \mathrm{O}_{2}\right)$ for $48 \mathrm{~h}$. The culture supernatants were collected and centrifuged at 2,000 x g for $20 \mathrm{~min}$ at $4^{\circ} \mathrm{C}$ to remove any large particles of debris. After an additional centrifugation at 100,000 x g for $1 \mathrm{~h}$ at $4^{\circ} \mathrm{C}$, the pellet was washed twice in PBS (26). The collected BMSC-EVs were then resuspended in $50 \mu \mathrm{l}$ PBS and stored at $-80^{\circ} \mathrm{C}$ for use in the subsequent experiments. The amount of BMSC-EVs was determined by measuring the total protein content using the Bradford method (Sigma-Aldrich; Merck KGaA).
Scanning electron microscopy. The EVs were suspension-fixed in $4 \%$ paraformaldehyde dissolved in PBS for $12 \mathrm{~h}$ at $4^{\circ} \mathrm{C}$. Subsequently, the EVs were dehydrated through a graded series of ethanol/water (50-100\%, v/v) solutions, and then dried at the critical point. The samples were gold-sputtered and examined under a Hitachi S-4800 field emission scanning electron microscope (Hitachi, Tokyo, Japan) at an acceleration voltage of $10 \mathrm{kV}$.

Characterization of the BMSC-EVs. The size distribution of the EVs was measured using a particle size analyzer (Nicomp 380 ZLS; Particle Sizing Systems, Port Richey, FL, USA) (27). The samples were illuminated by red laser light $(15 \mathrm{~mW}$, $635 \mathrm{~nm}$ ), and a digital camera captured the scattered light. The EVs were automatically tracked and sized, based on Brownian motion and their diffusion coefficient. The results are displayed as representative size distribution profiles.

A phenotypic profile of the EVs was determined using bead-based flow cytometry. Briefly, the BMSC-EVs were bound to aldehyde/sulfate latex beads (4 mm; Molecular Probes; Thermo Fisher Scientific, Inc.) suspended in 2-(N-morpholino) ethane sulfonic acid (MES) buffer $(0.025 \mathrm{M}$ MES, $0.154 \mathrm{M} \mathrm{NaCl}$; pH 6.0) overnight at $4^{\circ} \mathrm{C}$ with gentle agitation. The reaction was terminated by incubation for $30 \mathrm{~min}$ with $200 \mathrm{mM}$ glycine at room temperature, to saturate any remaining free binding sites on the beads. After two washes in PBS containing 4\% FBS, the BMSC-EVs-coated beads were stained with the following antibodies: Fluorescein isothiocyanate (FITC)-or phycoerythrin (PE)-conjugated anti-cluster of differentiation CD81 (catalog no. 559519, 1:400), CD63 (catalog no. 551458, 1:200), CD29 (catalog no. 555005, 1:200), CD90 (catalog no. 554894, 1:200), CD45 (catalog no. 559135, 1:200), CD31 (catalog no. 555026, 1:200) purchased from BD Biosciences (San Diego, CA, USA), with or without the following secondary antibodies: PE streptavidin; catalog no. 554061; 1:200 and goat anti-mouse 488; catalog no. A11001; 1:200, at $4^{\circ} \mathrm{C}$ overnight. Beads without BMSC-EVs were prepared to determine the forward and side scatter signals of the beads. All data were collected on an Epics Altra Hypersort ${ }^{\mathrm{TM}}$ system (Beckman Coulter, Inc., Brea, CA, USA) and were analyzed with FlowJo Software 7.6.1 (FlowJo, LLC, Ashland, OR, USA).

Cellular uptake of BMSC-EVs. BMSC-EVs were labeled with Cell Tracker ${ }^{\mathrm{TM}}$ CM-Dil (Thermo Fisher Scientific, Inc.). Briefly, $1 \mu \mathrm{l}$ cell-labeling solution was added to $200 \mu \mathrm{g} \mathrm{EVs}$ suspended in $1 \mathrm{ml} \mathrm{PBS}$, and was incubated for $20 \mathrm{~min}$ at $37^{\circ} \mathrm{C}$. Subsequently, the mixture was centrifuged at $100,000 \mathrm{x} \mathrm{g}$ for $1 \mathrm{~h}$ at $4^{\circ} \mathrm{C}$, the supernatants were removed, and the BMSC-EVs were gently resuspended in PBS. This washing procedure was repeated 3 times.

Human umbilical vein endothelial cells (HUVECs) were purchased from ScienCell Research Laboratories (Carlsbad, CA, USA), and were cultured in DMEM supplemented with $10 \% \mathrm{FBS}$ and $1 \%$ penicillin-streptomycin at $37^{\circ} \mathrm{C}$ in a humidified incubator $\left(5 \% \mathrm{CO}_{2}\right)$. Following incubation with CM-Dil-labeled BMSC-EVs $(20 \mu \mathrm{g} / \mathrm{ml})$ for 2,4 or $6 \mathrm{~h}$, the cells were washed twice with PBS, fixed in $4 \%$ paraformaldehyde and stained with DAPI. The cells were then observed under a confocal microscope (Leica TCS SP5; Leica Microsystems 
GmbH, Wetzlar Germany). The images were collected and merged using Image-Pro Plus 6 (Media Cybernetics, Inc., Rockville, MD, USA).

Cell viability. HUVECs $\left(1.0 \times 10^{3}\right.$ cells/well) were plated in 96 -well plates (3 wells/group) and treated with 50 or $100 \mu \mathrm{g} / \mathrm{ml}$ BMSC-EVs for 6 days. At the indicated time-points (1, 2, 3, 4 and 5 days), cell viability was determined using Cell Counting Kit-8 (CCK8; Dojindo Molecular Technologies, Inc., Kumamoto, Japan) according to the manufacturer's protocol. The absorbance was measured at $450 \mathrm{~nm}$ using an ELISA reader (Bio-Tek Instruments, Inc., Winooski, VT, USA). All experiments were repeated $\geq 3$ times.

Tube formation. Matrigel (BD Biosciences) was thawed and pipetted into a 24-well culture plate, and was incubated at $37^{\circ} \mathrm{C}$ for $30 \mathrm{~min}$ to allow solidification. HUVECs $\left(5 \times 10^{4}\right)$ were treated with 50 or $100 \mu \mathrm{g} / \mathrm{ml} \mathrm{BMSC-EVs}$ for $12 \mathrm{~h}$ at $37^{\circ} \mathrm{C}$. Tube formation was examined by phase-contrast microscopy (Olympus Corporation, Tokyo, Japan), and the number of network structures was quantified using Image-Pro Plus 6 (Media Cybernetics, Inc.). The data were statistically analyzed using GraphPad Prism 5 software (GraphPad Software, Inc., La Jolla, CA, USA). All experiments were repeated $\geq 3$ times.

Cell migration. Cell migration was assessed by scratch assays. Briefly, HUVECs were grown to confluence $\left(2 \times 10^{5}\right.$ cell $\left./ \mathrm{ml}\right)$ in a 6-well plate and a scratch was made in the cell field using a 1,000 $\mu \mathrm{l}$ pipette tip. The wells were washed with PBS to remove any debris and incubated with 50 or $100 \mu \mathrm{g} / \mathrm{ml}$ BMSC-EVs for $12 \mathrm{~h}$ at $37^{\circ} \mathrm{C}$. Bright-field microscopy images were then collected (Olympus Corporation) and cell migration was assessed using Image-Pro Plus 6 (Media Cybernetics, Inc.). The data were statistically analyzed using GraphPad Prism 5 software (GraphPad Software, Inc.). All experiments were repeated $\geq 3$ times.

Isolation and preparation of human fat tissue. During the period of June 2015 to November 2016, human liposuction aspirates were obtained from 10 healthy female donors aged from 28 to 40 who underwent liposuction of the abdomen at Shanghai 9th People's Hospital (Shanghai, China). Each patient provided written informed consent. The protocol for this study was approved by the Ethics Committee of Shanghai Jiao Tong University School of Medicine. Prior to initiation of the procedure, the liposuction site was infiltrated with tumescent solution containing $0.08 \%$ lidocaine and a 1: 500,000-unit dilution of epinephrine. Under sterile conditions, the lipid part of the fat aspirate was rinsed with sterile PBS to remove blood, saline and the local anesthetic. The adipose portion of the liposuction aspirate was placed in an upright position to obtain a clear separation of the fluids and oil. The upper (oil) and lower (blood and infiltration liquids) layers were removed, and the middle portion containing the fat particles was immediately transferred to $50 \mathrm{ml}$ centrifuge tubes and stored at $4^{\circ} \mathrm{C}$ until further use.

Animal model and study design. Female BALB/c-nu nude mice (age, 6 weeks; weight, 14-17 g) were obtained from the Shanghai Chuansha Experimental Animal Raising Farm
(Shanghai, China). Mice were kept at a temperature of $20-26^{\circ} \mathrm{C}$, a relative humidity of $40-70 \%$ and a day/night cycle of 12/12 h, with food and water ad libitum, and a total of 27 mice were randomly divided into three groups ( $\mathrm{n}=9$ mice/group). In the control (untreated) group, mice were injected with $1 \mathrm{ml}$ human fat and $200 \mu \mathrm{l}$ sterile PBS; in the low-dose EV group, mice were injected with $1 \mathrm{ml}$ human fat and $200 \mu \mathrm{l}$ sterile PBS containing $50 \mu \mathrm{g}$ BMSC-EVs; in the high-dose EV group, mice were injected with $1 \mathrm{ml}$ human fat and $200 \mu 1$ sterile PBS containing $100 \mu \mathrm{g}$ BMSC-EVs.

The scalp of the mouse was chosen as the recipient site for fat injection due to the absence of subcutaneous fat in this area. The mice were anesthetized with $0.2 \mathrm{ml} 5 \%$ chloral hydrate and a subcutaneous tunnel was created by antegradely passing a needle from the dorsalis to the scalp. Fat was then injected using a $14 \mathrm{G}$ needle in the retrograde direction while pulling the needle out. Following injection, the skin incisions were closed with \#6/0 non-absorbable sutures. A total of 12 weeks following transplantation, the mice were sacrificed and the grafts were harvested. Macroscopic inspection revealed that the fat grafts were surrounded by a thin envelope, making it easy to isolate them from the host tissue.

Graft volume. The harvested fat tissue grafts were weighed on a balance and their volumes were determined according to the liquid overflow method (13). Briefly, a graduated cylinder was filled with PBS, the fat tissues were immersed in the cylinder, and the fat volume was determined by the consequent increase in buffer volume.

Histology and immunohistochemistry. Following volume determination, the grafts removed from the scalp were fixed in $4 \%$ paraformaldehyde for $12 \mathrm{~h}$, embedded in paraffin, and cut into sections (size, $8 \mu \mathrm{m}$ ). These sections were stained with hematoxylin for 15-30 min and eosin for 3-5 $\mathrm{min}$ (H\&E stain) for structural analysis and Masson's staining (acid fuchsin stain for 5-10 $\mathrm{min}$ and aniline blue stain for $5 \mathrm{~min}$ ) for analysis of the fibrotic area. A total of 5 randomly selected fields from each sample ( $n=3$ per group) were imaged under an inverted microscope (Olympus Corporation).

Immunohistochemical staining was performed according a method previously published (25). In brief, paraffin-embedded sections ( $8 \mathrm{~mm}$ thick) were incubated with goat anti-CD31 antibody (catalog no. sc-1506; Santa Cruz Biotechnology, Inc., Dallas, TX, USA) overnight at $4^{\circ} \mathrm{C}$, followed by incubation with a horseradish peroxidase-conjugated rabbit anti-goat secondary antibody (catalog no. E0466; Dako; Agilent Technologies, Inc.) at room temperature for $30 \mathrm{~min}$, and colorization with 3,3'-diaminobenzidine tetrahydrochloride (Dako; Agilent Technologies, Inc.) at room temperature for $3 \mathrm{~min}$. A total of 5 randomly selected fields from each sample ( $n=3$ /group) were imaged under a light microscope (Nikon Eclipse 90i; Nikon Corporation, Tokyo, Japan). The number of CD31-positive capillaries was calculated using Image-Pro Plus 6 (Media Cybernetics, Inc.), and the data were statistically analyzed using GraphPad Prism 5 software (GraphPad Software, Inc.) (25).

Reverse transcription-quantitative polymerase chain reaction $(R T-q P C R)$. Total RNA from the control and low-dose EV 
Table I. Primers used in quantitative polymerase chain reaction analysis.

\begin{tabular}{lcl}
\hline Gene & Primer size (bp) & \multicolumn{1}{c}{ Primer sequences } \\
\hline PDGF-A & 199 & F:GCAAGACCAGGACGGTCATTTAC \\
PDGFR-A & 193 & R:GGCTTCTTCCTGACATACTC \\
FEGF-A & 130 & R:CCATGCAGTTGCCTTACGAC \\
& & F:GGAGATCCCTTCGTCACTAGACC \\
TGF- $\beta$ & 226 & R:GGCGATTAGCAGCAGATATAAGAAA \\
& & F:AACCCCCATTGCTGTCCCGTG \\
GAPDH & 235 & R:GCGCTGAATCGAAAGCCTGT \\
& & F:GACTTCAACAGCAACTCCCAC \\
& & R:TCCACCACCCTGTTCTGTA
\end{tabular}

PDGF-A, platelet-derived growth factor $\alpha$; PDGFR-A, platelet-derived growth factor receptor $\alpha$; VEGF-A, vascular endothelial growth factor A; TGF- $\beta$, transforming growth factor $\beta$; F, forward; R, reverse.

groups were extracted and reverse transcribed to obtain cDNA, as previously described (25). RT-qPCR was performed using a Power SYBR-Green PCR Master Mix (Applied Biosystems; Thermo Fisher Scientific, Inc.) in an Mx3000P qPCR system thermal cycler (Stratagene; Agilent Technologies, Inc., Santa Clara, CA, USA). Cycling conditions were as follows: An initial predenaturation step at $95^{\circ} \mathrm{C}$ for $10 \mathrm{~min}$, followed by 40 cycles of denaturation at $95^{\circ} \mathrm{C}$ for $30 \mathrm{sec}$, annealing at $60^{\circ} \mathrm{C}$ for $30 \mathrm{sec}$ and extension at $72^{\circ} \mathrm{C}$ for $45 \mathrm{sec}$. The primers used for detection of the following proangiogenic factors: Platelet-derived growth factor $\alpha$ (PDGF-A), platelet-derived growth factor receptor $\alpha$ (PDGFR-A), vascular endothelial growth factor $\mathrm{A}$ (VEGF-A) and transforming growth factor $\beta$ (TGF- $\beta$ ) are listed in Table I. Gene expression was normalized to GAPDH expression according to the $2^{-\triangle \Delta \mathrm{Cq}}$ method (28). Each sample was tested in triplicate, and 3 independent experiments were performed.

Statistical analysis. The data were statistically analyzed using GraphPad Prism 5 software (GraphPad Software, Inc., La Jolla, CA, USA). Data are expressed as the mean \pm standard deviation. The significance of differences between groups was assessed by Student's t-test or one-way analysis of variance followed by the Newman-Keuls post hoc test. $\mathrm{P}<0.05$ was considered to indicate a statistically significant difference.

\section{Results}

Characterization of BMSC-EVs. To determine whether co-transplantation of fat with BMSC-EVs improves fat graft retention, EVs were isolated from the supernatants of rat BMSCs and were characterized. Scanning electron microscopy demonstrated that the EVs were heterogeneous with a spheroid-shaped morphology (Fig. 1A). Particle size analysis indicated that the average EV diameter was $228.4 \pm 28.8 \mathrm{~nm}$, with a range between 40 and $300 \mathrm{~nm}$ (Fig. 1B), thus suggesting that the EVs comprised a mixture of exosomes and microvesicles. Flow cytometry demonstrated that the EVs expressed high levels of the following BMSC-positive and EV-positive markers: CD90, CD29, CD81 and CD63, but not of the endothelial and hematopoietic cell markers: CD31 and CD45 (Fig. 1C and D). These findings indicated that the EVs were of BMSC origin.

Cellular uptake of BMSC-EVs. In order to determine whether the BMSC-EVs interact with vascular endothelial cells, HUVECs were incubated with CM-Dil-labeled BMSC-EVs and observed by confocal microscopy. Following a 2-h incubation, CM-Dil was observed on the cell surface. With increasing incubation time, more fluorescent dye accumulated in the cytoplasm, eventually reaching a maximal signal at $6 \mathrm{~h}$ (Fig. 2). These results indicated that the EVs were internalized by HUVECs.

BMSC-EVs promote viability, migration and tube formation of HUVECs. To determine the effects of BMSC-EVs on endothelial cells, HUVECs were exposed to 50 or $100 \mu \mathrm{g} / \mathrm{ml}$ BMSC-EVs, and their cell viability, migration and tube-forming capabilities were assessed. As presented in Fig. 3A, $100 \mu \mathrm{g} / \mathrm{ml}$ EVs increased cell viability by $\sim 1.3$-fold compared with the control group after 5 days; however, there were no significant differences between the low $(50 \mu \mathrm{g} / \mathrm{ml})$ and high dose $(100 \mu \mathrm{g} / \mathrm{ml})$ treatment groups. An in vitro tube formation assay was conducted to assess the angiogenic effects of BMSC-EVs; the results demonstrated that more vessel-like structures were observed following EV treatment, in a dose-dependent manner (Fig. 3B and C). Quantitative and statistical analysis demonstrated that the high dose EV-treated HUVECs formed 3,419 \pm 97 vessel-like structures per section, whereas the control cells formed 2,553 \pm 107 , thus suggesting a 1.34 -fold increase in vessel-like structures following EV treatment (Fig. 3C). In addition, the cell migration assay indicated that treatment of HUVECs with high dose BMSC-EVs resulted in rapid closure of the scratched area, which was 1.39-fold faster compared with in the non-treated HUVECs; this effect was dose-dependent (Fig. 3D and E). These results suggested that BMSC-EVs may promote endothelial cell viability, migration and tube-forming capabilities in vitro, indicating that BMSC-EVs may exert angiogenesis-promoting activity. 

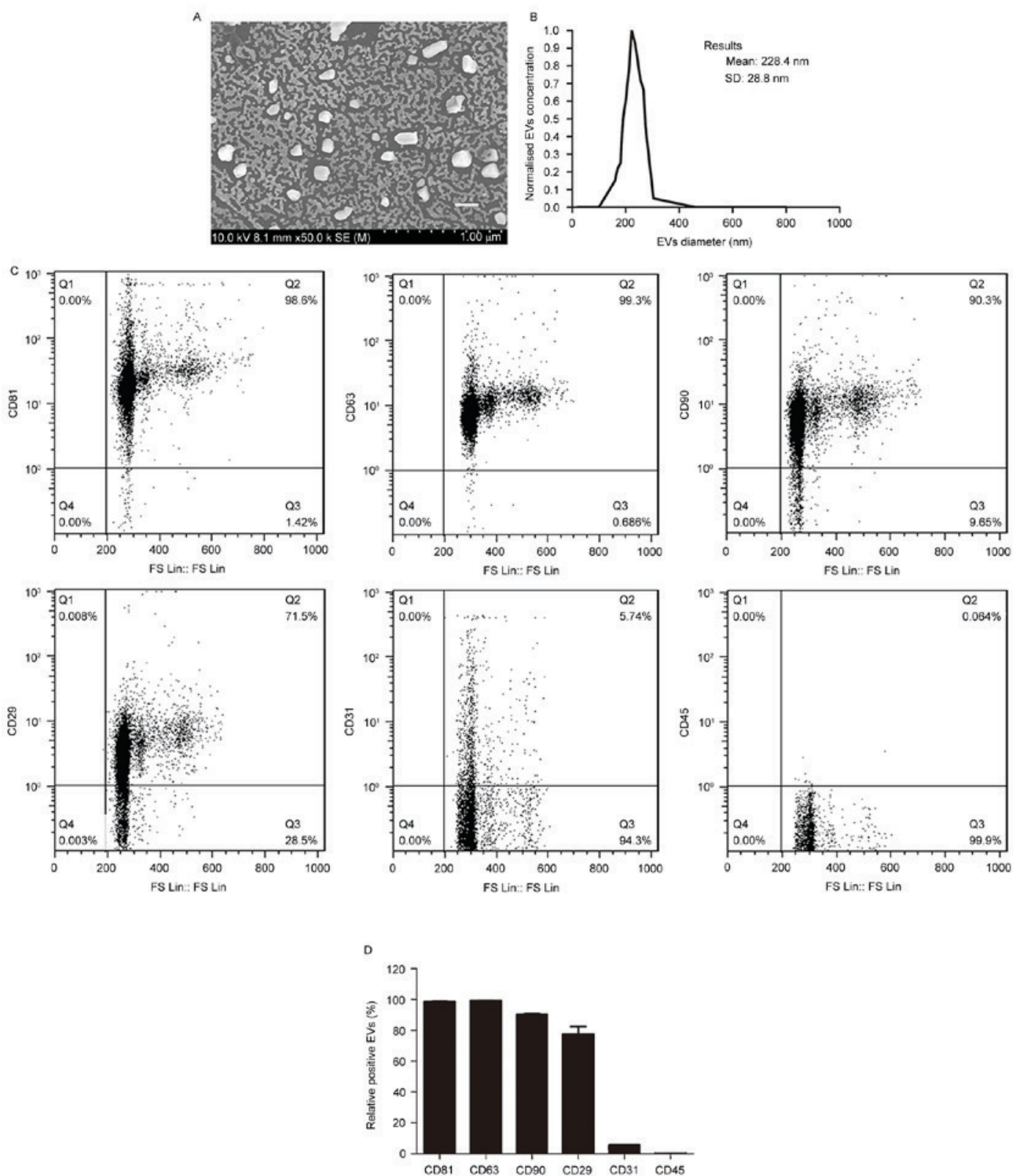

Figure 1. Characterization of bone marrow mesenchymal stem cell-derived EVs. (A) Morphological characteristics of the EVs, as revealed by scanning electron microscopy. Scale bar, $200 \mathrm{~nm}$. (B) Size distribution of the EVs. (C) Surface profile of EVs, as determined by flow cytometry. Representative results from 3 independent experiments are presented. (D) Quantitative analysis of the flow cytometry results (n=3/group). EVs, extracellular vesicles; CD, cluster of differentiation.

BMSC-EVs improve fat graft retention. To evaluate the effects of BMSC-EVs on the retention of transplanted fat grafts in animals, human fat prepared from liposuction aspirates was co-injected with BMSC-EVs into nude mice. A total of 12 weeks post-transplantation, all 27 mice in the control, low-dose EV and high-dose EV groups survived. No inflammation or abscesses were observed in the surgical areas. Subsequently, the mice were sacrificed and the fat grafts were harvested. A total of 5 representative images of grafts from each group $(n=9)$ are presented in Fig. 4A. The grafts in the $\mathrm{EV}$-treated groups appeared larger than those in the non-treated group (Fig. 4A). Statistical analysis (n=9/group) demonstrated that average fat volume in the control, low-dose EV and high-dose EV groups was $0.2018 \pm 0.0258,0.3283 \pm 0.0368$ and $0.3689 \pm 0.0241 \mathrm{ml}$, respectively, whereas the average fat weight in these groups was $0.1902 \pm 0.0252,0.3166 \pm 0.0363$ and $0.3297 \pm 0.0228 \mathrm{~g}$, respectively. These results suggested that EV treatment increased graft retention (Fig. 4B and C).

The graft structure was further analyzed by $\mathrm{H} \& \mathrm{E}$ and Masson staining after sectioning. As presented in Fig. 5, all of the grafts contained intact adipocytes, fibrous tissue and vacuoles. The grafts from the EV-treated groups had more intact and nucleated adipocytes with relatively fewer fibers and vacuoles, whereas more vacuoles were observed in the control group. No differences were observed between the low- and high-dose groups. These observations suggested that BMSC-EVs may enhance adipose tissue regeneration post-transplantation. 


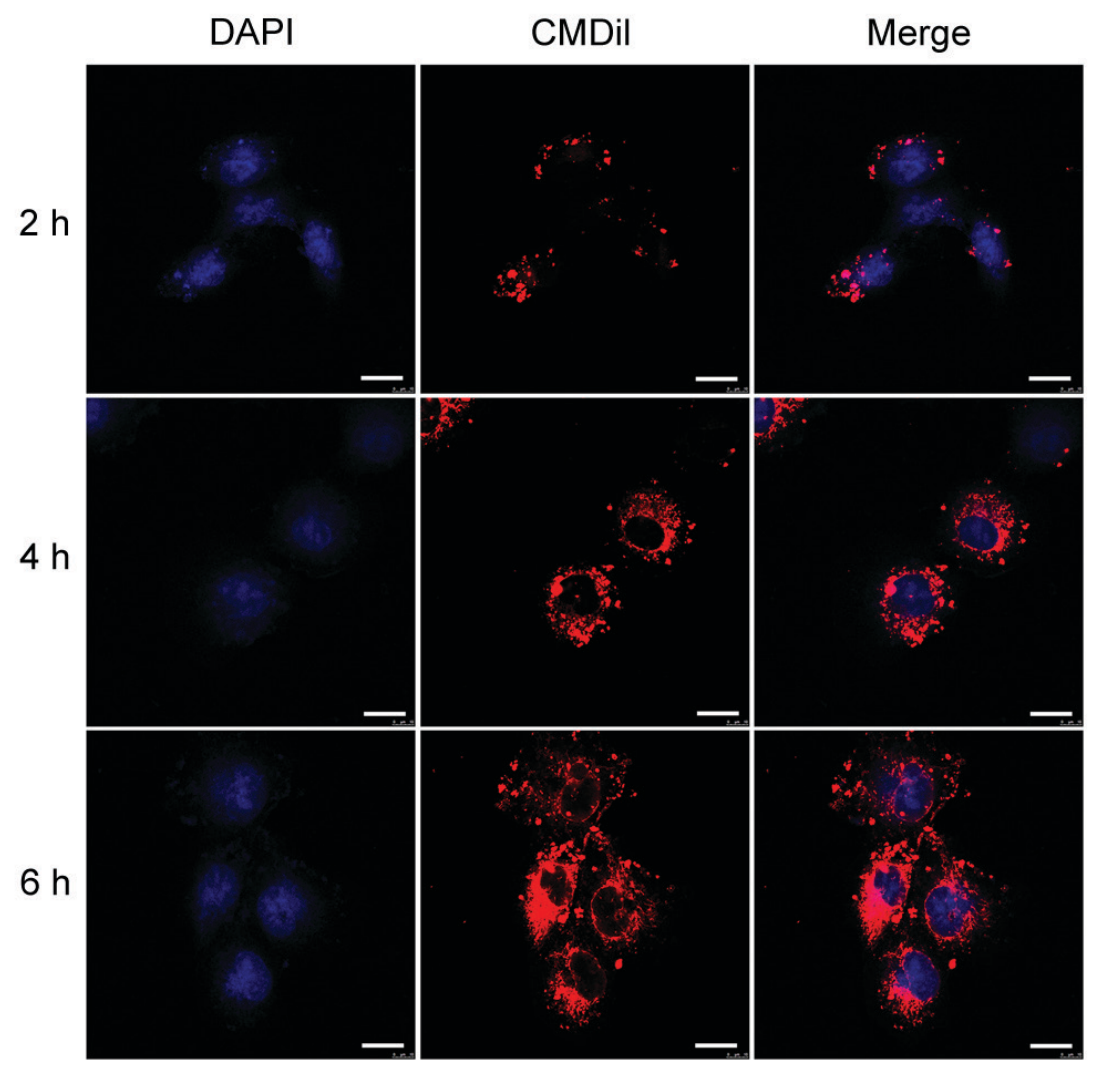

Figure 2. Internalization of BMSC-EVs by HUVECs. HUVECs were incubated with CM-Dil (red)-labeled BMSC-EVs for 2, 4 and 6 h. Cell nuclei were stained with DAPI (blue). Scale bar, $20 \mu \mathrm{m}$. BMSC-EVs, bone marrow mesenchymal stem cell-derived extracellular vesicles; HUVECs, human umbilical vein endothelial cells.

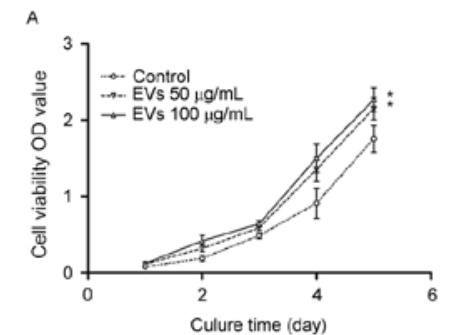

B
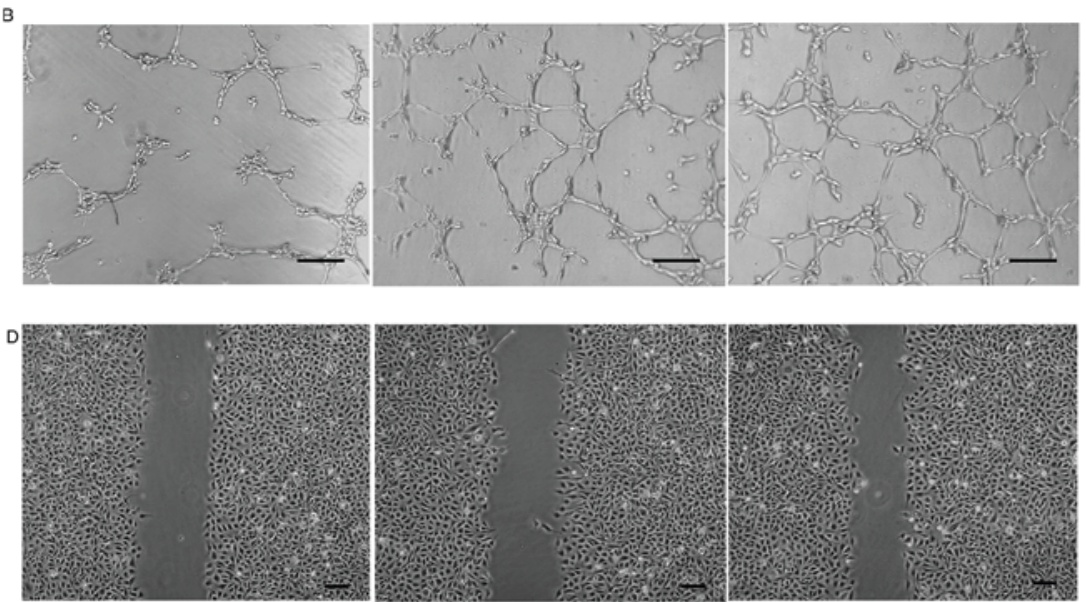
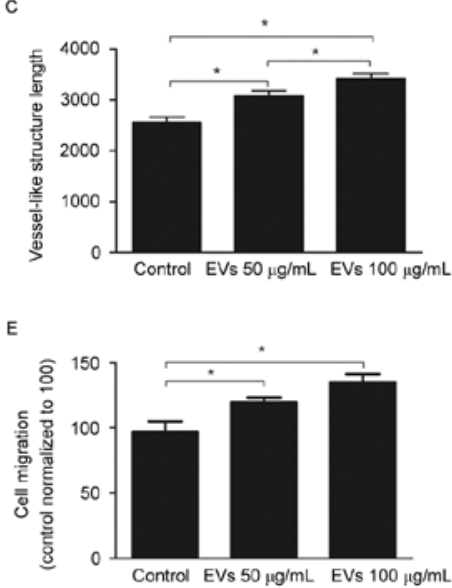

Figure 3. BMSC-EVs increased HUVEC viability, tube formation and migration in vitro. (A) Viability of HUVECs in the absence (control) or presence of two concentrations of BMSC-EVs, as determined by Cell Counting Kit-8 assay. (B) Tube formation of HUVECs in the absence (control) or presence of two doses of BMSC-EVs. Scale bar, $200 \mu \mathrm{m}$. (C) Quantitative analysis of the vessel-like structures formed in response to the two doses of BMSC-EVs. (D) Cell migration of HUVECs in the absence (control) or presence of two concentrations of BMSC-EVs. Scale bar, $200 \mu \mathrm{m}$. (E) Quantitative analysis of the recovered area after a scratch test in response to two doses of BMSC-EVs (n=9/group). "P<0.05. BMSC-EVs, bone marrow mesenchymal stem cell-derived extracellular vesicles; HUVECs, human umbilical vein endothelial cells; OD, optical density. 


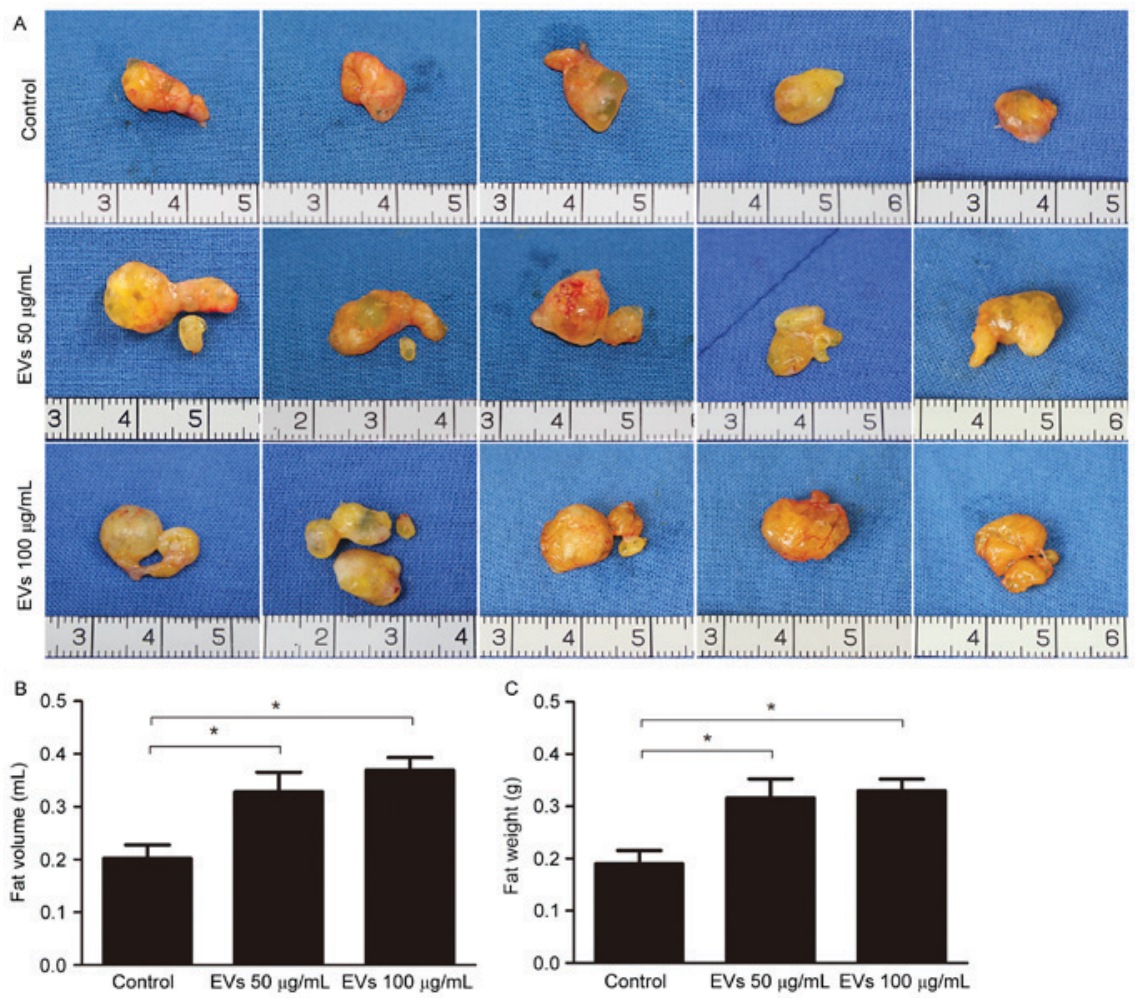

Figure 4. Bone marrow mesenchymal stem cell-derived extracellular vesicles improved fat graft retention. (A) Macroscopic images of the harvested fat grafts 12 weeks post-transplantation. (B) Quantitative analysis of the tissue volume of the fat grafts ( $\mathrm{n}=9 / \mathrm{group}$ ). (C) Quantitative analysis of the wet weight of the fat grafts (n=9/group). ${ }^{*} \mathrm{P}<0.05$

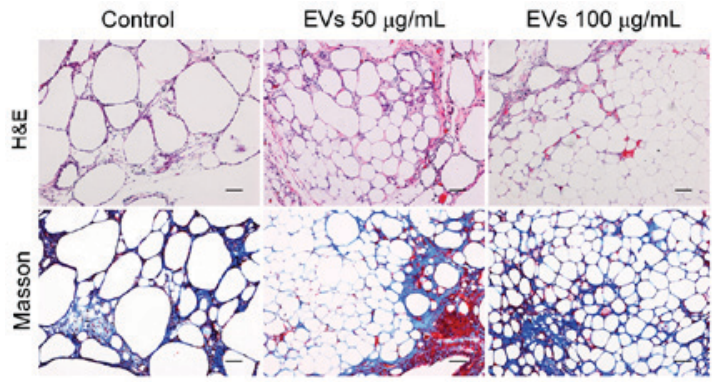

Figure 5. Histological analysis of fat grafts by H\&E and Masson trichrome staining. Scale bar, $100 \mu \mathrm{m}$. H\&E, hematoxylin and eosin.

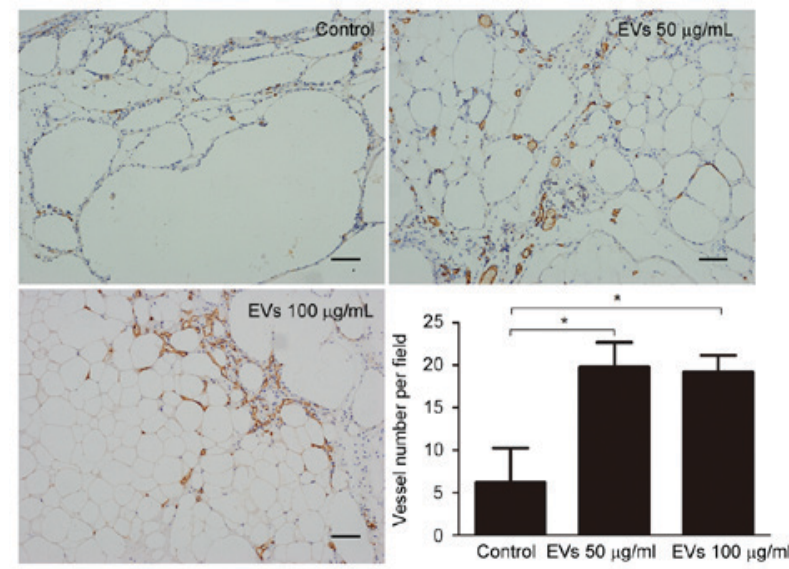

Figure 6. Angiogenesis was evaluated by anti-cluster of differentiation 31 staining. Grafts from the EV-treated groups exhibited significantly increased numbers of vessels compared with in the control group. Scale bar, $100 \mu \mathrm{m}$. ${ }^{*} \mathrm{P}<0.05$. EVs, extracellular vesicles.
BMSC-EVs promote fat retention through neovascularization. In the animal model, BMSC-EVs appeared to improve graft retention by promoting adipose tissue regeneration; therefore, the present study examined whether BMSC-EVs may facilitate neovascularization, which is an indispensable process for tissue regeneration. To detect CD31-positive capillaries, harvested fat tissue grafts were subjected to immunohistochemical staining with an anti-CD31 antibody. An increased number of CD31-positive capillaries was observed in the EV-treated groups compared with in the control group (Fig. 6). Capillary density (vessel number per field) was calculated from 5 randomly selected fields in each sample from the control, low-dose EV and high-dose EV groups; capillary density was $6.20 \pm 4.07,19.80 \pm 2.91$ and $19.20 \pm 1.99$, respectively, indicating that treatment with EVs may promote neovascularization (Fig. 6). Subsequently, the gene expression levels of proangiogenic factors were analyzed in the fat grafts by RT-qPCR. The expression levels of PDGF-A (3.306 \pm 0.65 -fold), PDGFR-A (4.601 \pm 0.73 -fold), VEGF-A (1.970 \pm 0.16 -fold), and TGF- $\beta$ (10.310 \pm 2.49 -fold) were increased in the $50 \mu \mathrm{g} / \mathrm{ml} \mathrm{EV}$-treated group compared with in the control group (Fig. 7). These results suggested that BMSC-EVs may promote neovascularization by stimulating the secretion of proangiogenic factors.

\section{Discussion}

Free fat grafting is an advantageous technique that may be used as a contouring tool in reconstructive and cosmetic surgical procedures; however, its use is limited by the unpredictable and poor long-term retention of the transplanted fat (4). Although 

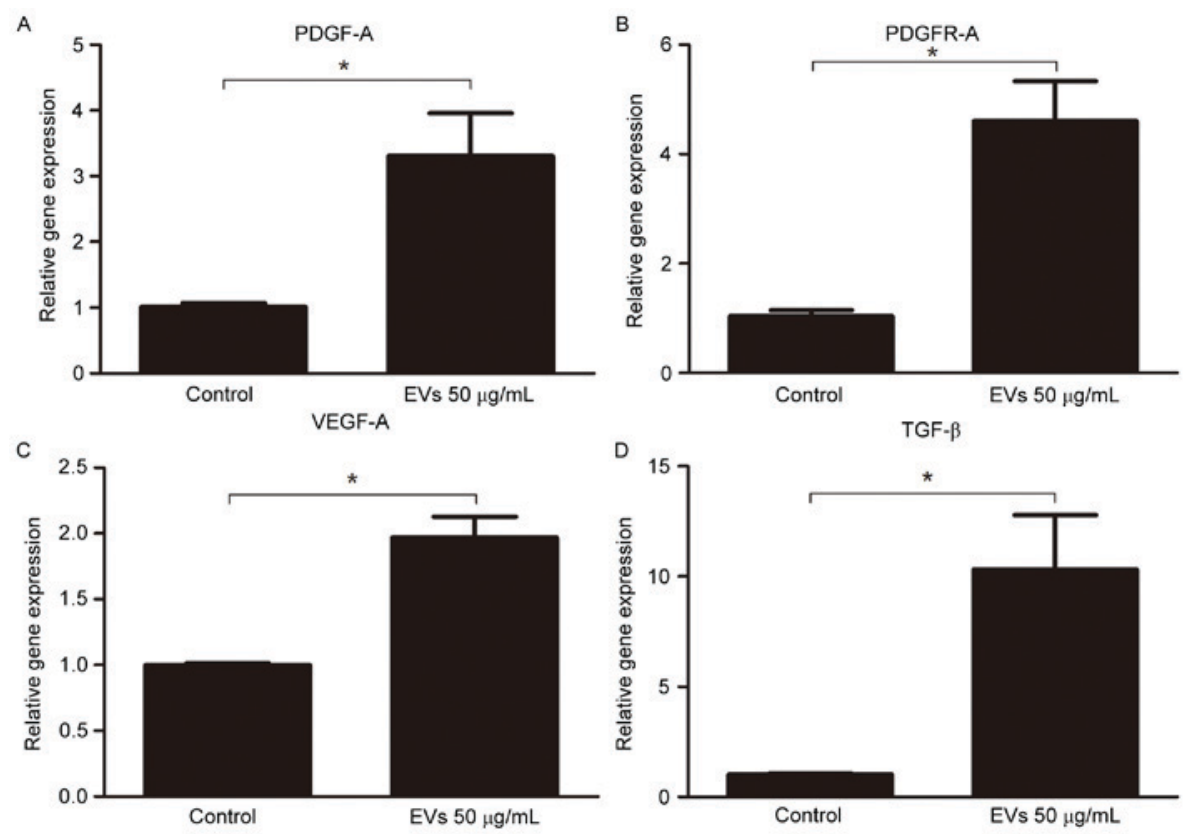

Figure 7. Expression of the proangiogenic growth factors (A) PDGF-A, (B) PDGFR-A, (C) VEGF-A and (D) TGF- $\beta$. in fat grafts, as determined by reverse transcription-quantitative polymerase chain reaction. Each sample was tested in triplicate ( $\mathrm{n}=3 / \mathrm{group}$ ). EVs, extracellular vesicles; PDGF-A, platelet-derived growth factor $\alpha$; PDGFR-A, platelet-derived growth factor receptor $\alpha$; VEGF-A, vascular endothelial growth factor A; TGF- $\beta$, transforming growth factor $\beta$. "P<0.05.

numerous methods for improving fat graft survival have been investigated, further improvements are still required.

At present, the exact mechanisms underlying fat graft survival remain unclear. One generally accepted mechanism leading to graft loss is a lack of adequate revascularization of the fat post-transplantation. Karacaoglu et al (9) reported that fat graft survival was greatest in the supramuscular layer, and indicated that fat grafts used in relatively more vascular areas underwent lower rates of resorption. Eto et al (7) demonstrated that adipocytes died as early as the first day after ischemia, endothelial cells died second, and finally adipose-derived stromal cells (ADSCs) died on day 3. Furthermore, Aygit et al (29) revealed that revascularization of fat grafts occurred $48 \mathrm{~h}$ post-transplantation, indicating that it is too late for the survival of adipocytes. Various approaches for accelerating angiogenesis have been successfully undertaken to enhance fat survival post-transplantation, including the administration of basic fibroblast growth factors, interleukin- 8 and erythropoietin $(11,30,31)$; VEGF-based gene therapy $(8,32)$; and endothelial cell and MSC therapies $(13,16,33)$.

EVs released by MSCs have recently been reported to exert proangiogenic effects in numerous ischemic animal models $(18,34)$. The present study demonstrated that BMSC-EVs stimulated neovascularization and improved retention of transplanted fat grafts in a nude mice model. The present results confirmed that EVs were of BMSC origin, as the cells expressed high levels of the BMSC-positive markers, CD81, CD63, CD90 and CD29, and were negative for the endothelial and hematopoietic cell markers, CD31 and CD45 (Fig. 1C and D). Confocal microscopy demonstrated that the BMSC-EVs interacted with endothelial cells in vitro (Fig. 2). Furthermore, the BMSC-EVs improved HUVEC in vitro cell viability, migration and tube formation, demonstrating the proangiogenic potential of BMSC-EVs (Fig. 3). Based on these findings, the effects of BMSC-EVs on free fat grafts were investigated in an animal model. The grafts from the EV-treated groups had higher tissue volume and weight, and improved histology, indicating a better overall survival than those in the control group at 12 weeks post-transplantation (Figs. 4 and 5). Immunohistochemical and RT-qPCR analyses also supported the hypothesis that the observed improvement in retention of fat graft weight and volume was attributable to the induction of angiogenesis (Figs. 6 and 7). These observations are also in agreement with the findings of previous studies, demonstrating a link between neovascularization and improved fat graft survival $(8,16,35)$.

In addition to neovascularization, the importance of adipogenesis for long-term retention of transplanted fat has been implicated. Previous in vivo studies have demonstrated that the majority of adipocytes in free grafts die shortly after transplantation, whereas only ADSCs survive $(7,36,37)$. In the 'three zone' theory suggested by Yoshimura et al (38), the survival of fat grafts is largely dependent on adipose tissue regeneration post-transplantation, and CD34-positive cells are very likely the seed cells for adipogenic progression. Therefore, recent studies $(14,39)$ have focused on the therapeutic effects of adipose-derived cells, including stromal vascular fraction and ADSCs, on fat grafts. These cells may improve tissue outcomes by increasing the vascularity and the secretion of growth factors that improve tissue survival. Yoshimura et al (40) reported that ADSCs are able to enhance angiogenesis and improve the survival rate of non-vascularized grafted fat. In addition, these cells may function as seed cells for adipogenesis. Compared with these cell-based therapies, BMSC-EVs serve a proangiogenic role, but do not undergo adipocyte differentiation. However, the present study demonstrated that the improvements in fat 
graft retention achieved by BMSC-EVs were as good as those from cell-based therapies $(16,39)$, indicating that revascularization is necessary for free fat grafting. This finding is in agreement with a recent study, which suggested that ADSCs are involved in the process of graft revascularization via paracrine action, but do not undergo significant adipogenic differentiation (41).

With respect to clinical implications, cross-species MSCs have been successfully used in various in vivo animal models. In the nude mice model system used in the present study, human fat with EVs isolated from rat BMSCs was transplanted into the mice. In addition, Li et al (42) identified 94 reports of in vivo cross-species administration of MSCs in various experimental models. Although the majority of these studies involved human MSCs, pig, rat or guinea pig MSCs were also occasionally used. A total of $93.6 \%$ of the cases confirmed that the MSCs were successfully engrafted and functioned well in the different species, with only $6.4 \%$ of cases reporting failure. In the present study, rat BMSC-EVs were internalized by HUVECs and promoted their viability, migration and tube-forming capabilities in vitro. In the nude mice experiments, the results indicated that rat BMSC-EVs improved the retention of human fat grafts, which suggests that BMSC-EVs function across species barriers, in accordance with a previous report that rat MSCs prolonged xenogenic skin graft survival in mice (43).

In conclusion, the present study is the first, to the best of our knowledge, to demonstrate that co-transplantation of fat grafts with BMSC-EVs improves the long-term retention and transplanted fat quality in a mouse model. In addition, the present findings suggested that this beneficial effect is likely mediated by the proangiogenic effects of BMSC-EVs. These results suggested that effective employment of BMSC-EVs may have potential as a therapeutic option in fat transplantation. However, the detailed mechanisms underlying the function of BMSC-EVs remain to be investigated.

\section{Acknowledgements}

The present study was supported by the Major State Basic Research Development Program of China (grant no. 2011CB964704) and the National Natural Science Foundation of China (grant nos. 81271714 and 31170944).

\section{References}

1. Atik B, Oztürk G, Erdoğan E and Tan O: Comparison of techniques for long-term storage of fat grafts: An experimental study. Plast Reconstr Surg 118: 1533-1537, 2006.

2. Dong Z, Peng Z, Chang Q, Zhan W, Zeng Z, Zhang S and Lu F: The angiogenic and adipogenic modes of adipose tissue after free fat grafting. Plast Reconstr Surg 135: 556e-567e, 2015.

3. Mizoguchi T, Kijima Y, Hirata M, Kaneko K, Arima H, Nakajo A, Higashi M, Tabata K, Koriyama C, Arigami T, et al: Histological findings of an autologous dermal fat graft implanted onto the pectoralis major muscle of a rat model. Breast Cancer 22: 578-585, 2015.

4. Zhu M, Zhou Z, Chen Y, Schreiber R, Ransom JT, Fraser JK, Hedrick MH, Pinkernell K and Kuo HC: Supplementation of fat grafts with adipose-derived regenerative cells improves long-term graft retention. Ann Plast Surg 64: 222-228, 2010.

5. Wetterau M, Szpalski C, Hazen A and Warren SM: Autologous fat grafting and facial reconstruction. J Craniofac Surg 23: 315-318, 2012.
6. Herold C, Ueberreiter K, Busche MN and Vogt PM: Autologous fat transplantation: Volumetric tools for estimation of volume survival. A systematic review. Aesthetic Plast Surg 37: 380-387, 2013.

7. Eto H, Kato H, Suga H, Aoi N, Doi K, Kuno S and Yoshimura K: The fate of adipocytes after nonvascularized fat grafting: Evidence of early death and replacement of adipocytes. Plast Reconstr Surg 129: 1081-1092, 2012.

8. Lu F, Li J, Gao J, Ogawa R, Ou C, Yang B and Fu B: Improvement of the survival of human autologous fat transplantation by using VEGF-transfected adipose-derived stem cells. Plast Reconstr Surg 124: 1437-1446, 2009.

9. Karacaoglu E, Kizilkaya E, Cermik H and Zienowicz R: The role of recipient sites in fat-graft survival: Experimental study. Ann Plast Surg 55: 63-68, 2005.

10. Yamaguchi M, Matsumoto F, Bujo H, Shibasaki M, Takahashi K, Yoshimoto S, Ichinose M and Saito Y: Revascularization determines volume retention and gene expression by fat grafts in mice. Exp Biol Med (Maywood) 230: 742-748, 2005.

11. Hamed S, Egozi D, Kruchevsky D, Teot L, Gilhar A and Ullmann Y: Erythropoietin improves the survival of fat tissue after its transplantation in nude mice. PLoS One 5: e13986, 2010.

12. Hong SJ, Lee JH, Hong SM and Park CH: Enhancing the viability of fat grafts using new transfer medium containing insulin and beta-fibroblast growth factor in autologous fat transplantation. J Plast Reconstr Aesthet Surg 63: 1202-1208, 2010.

13. Yi C, Pan Y, Zhen Y, Zhang L, Zhang X, Shu M, Han Y and Guo S: Enhancement of viability of fat grafts in nude mice by endothelial progenitor cells. Dermatol Surg 32: 1437-1443, 2006.

14. Seyhan N, Alhan D, Ural AU, Gunal A, Avunduk MC and Savaci N: The effect of combined use of platelet-rich plasma and adipose-derived stem cells on fat graft survival. Ann Plast Surg 74: 615-620, 2015.

15. Zhu M, Dong Z, Gao J, Liao Y, Xue J, Yuan Y, Liu L, Chang Q and Lu F: Adipocyte regeneration after free fat transplantation: Promotion by stromal vascular fraction cells. Cell Transplant 24: 49-62, 2015.

16. Zhao J, Yi C, Zheng Y, Li L, Qiu X, Xia W, Su Y, Diao J and Guo $S$ : Enhancement of fat graft survival by bone marrow-derived mesenchymal stem cell therapy. Plast Reconstr Surg 132: 1149-1157, 2013.

17. See F, Seki T, Psaltis PJ, Sondermeijer HP, Gronthos S, Zannettino AC, Govaert KM, Schuster MD, Kurlansky PA, Kelly DJ, et al: Therapeutic effects of human STRO-3-selected mesenchymal precursor cells and their soluble factors in experimental myocardial ischemia. J Cell Mol Med 15: 2117-2129, 2011.

18. Bian S, Zhang L, Duan L, Wang X, Min Y and Yu H: Extracellular vesicles derived from human bone marrow mesenchymal stem cells promote angiogenesis in a rat myocardial infarction model. J Mol Med (Berl) 92: 387-397, 2014.

19. Tögel F, Weiss K, Yang Y, Hu Z, Zhang P and Westenfelder C: Vasculotropic, paracrine actions of infused mesenchymal stem cells are important to the recovery from acute kidney injury. Am J Physiol Renal Physiol 292: F1626-F1635, 2007.

20. Vader P, Breakefield XO and Wood MJ: Extracellular vesicles: Emerging targets for cancer therapy. Trends Mol Med 20: 385-393, 2014.

21. van der Pol E, Böing AN, Harrison P, Sturk A and Nieuwland R: Classification, functions, and clinical relevance of extracellular vesicles. Pharmacol Rev 64: 676-705, 2012.

22. Tetta C, Bruno S, Fonsato V, Deregibus MC and Camussi G: The role of microvesicles in tissue repair. Organogenesis 7: 105-115, 2011.

23. Reis LA,BorgesFT,Simões MJ,Borges AA, Sinigaglia-Coimbra R and Schor N: Bone marrow-derived mesenchymal stem cells repaired but did not prevent gentamicin-induced acute kidney injury through paracrine effects in rats. PLoS One 7: e44092, 2012.

24. Lai RC, Chen TS and Lim SK: Mesenchymal stem cell exosome: A novel stem cell-based therapy for cardiovascular disease. Regen Med 6: 481-492, 2011.

25. Lian J, Lu Y, Xu P, Ai A, Zhou G, Liu W, Cao Y and Zhang WJ: Prevention of liver fibrosis by intrasplenic injection of high-density cultured bone marrow cells in a rat chronic liver injury model. PLoS One 9: e103603, 2014.

26. Deregibus MC, Cantaluppi V, Calogero R, Lo Iacono M, Tetta C, Biancone L, Bruno S, Bussolati B and Camussi G: Endothelial progenitor cell derived microvesicles activate an angiogenic program in endothelial cells by a horizontal transfer of mRNA. Blood 110: 2440-2448, 2007. 
27. Osterman CJ, Lynch JC, Leaf P, Gonda A, Ferguson Bennit HR, Griffiths D and Wall NR: Curcumin modulates pancreatic adenocarcinoma cell-derived exosomal function. PLoS One 10 e0132845, 2015.

28. Livak KJ and Schmittgen TD: Analysis of relative gene expression data using real-time quantitative PCR and the 2(-Delta Delta C(T)) method. Methods 25: 402-408, 2001.

29. Aygit AC, Sarikaya A, Doganay L, Top H, Cakir B and Firat MF: The fate of intramuscularly injected fat autografts: An experimental study in rabbits. Aesthet Plast Surg 28: 334-339, 2004.

30. Jiang A, Li M, Duan W, Dong Y and Wang Y: Improvement of the survival of human autologous fat transplantation by adipose-derived stem-cells-assisted lipotransfer combined with bFGF. ScientificWorld Journal 2015: 968057, 2015.

31. Shoshani O, Livne E, Armoni M, Shupak A, Berger J, Ramon Y, Fodor L, Gilhar A, Peled IJ and Ullmann Y: The effect of interleukin- 8 on the viability of injected adipose tissue in nude mice. Plast Reconstr Surg 115: 853-859, 2005.

32. Yi CG, Xia W, Zhang LX, Zhen Y, Shu MG, Han Y and Guo SZ: VEGF gene therapy for the survival of transplanted fat tissue in nude mice. J Plast Reconstr Aesthet Surg 60: 272-278, 2007.

33. Ko MS, Jung JY, Shin IS, Choi EW, Kim JH, Kang SK and Ra JC: Effects of expanded human adipose tissue-derived mesenchymal stem cells on the viability of cryopreserved fat grafts in the nude mouse. Int J Med Sci 8: 231-238, 2011.

34. Xin H, Li Y, Cui Y, Yang JJ, Zhang ZG and Chopp M: Systemic administration of exosomes released from mesenchymal stromal cells promote functional recovery and neurovascular plasticity after stroke in rats. J Cereb Blood Flow Metab 33: 1711-1715, 2013.

35. Sezgin B, Ozmen S, Bulam H, Omeroglu S, Yuksel S, Cayci B and Peker T: Improving fat graft survival through preconditioning of the recipient site with microneedling. J Plast Reconstr Aesthet Surg 67: 712-720, 2014.
36. Dong Z, Peng Z, Chang Q and Lu F: The survival condition and immunoregulatory function of adipose stromal vascular fraction (SVF) in the early stage of nonvascularized adipose transplantation. PLoS One 8: e80364, 2013.

37. Sunaga A, Sugawara Y, Katsuragi-Tomioka Y and Kobayashi E: The fate of nonvascularized fat grafts: Histological and bioluminescent study. Plast Reconstr Surg Glob Open 1: e40, 2013.

38. Yoshimura K, Eto H, Kato H, Doi K and Aoi N: In vivo manipulation of stem cells for adipose tissue repair/reconstruction. Regen Med 6 (6 Suppl): S33-S41, 2011.

39. Piccinno MS, Veronesi E, Loschi P, Pignatti M, Murgia A, Grisendi G, Castelli I, Bernabei D, Candini O, Conte P, et al: Adipose stromal/stem cells assist fat transplantation reducing necrosis and increasing graft performance. Apoptosis 18: 1274-1289, 2013.

40. Yoshimura K, Sato K, Aoi N, Kurita M, Inoue K, Suga H, Eto H, Kato H, Hirohi T and Harii K: Cell-assisted lipotransfer for facial lipoatrophy: Efficacy of clinical use of adipose-derived stem cells. Dermatol Surg 34: 1178-1185, 2008.

41. Garza RM, Rennert RC, Paik KJ, Atashroo D, Chung MT, Duscher D, Januszyk M, Gurtner GC, Longaker MT and Wan DC: Studies in fat grafting: Part IV. Adipose-derived stromal cell gene expression in cell-assisted lipotransfer. Plast Reconstr Surg 135: 1045-1055, 2015.

42. Li J, Ezzelarab MB and Cooper DK: Do mesenchymal stem cells function across species barriers? Relevance for xenotransplantation. Xenotransplantation 19: 273-285, 2012.

43. Moadsiri A, Polchert D, Genrich K, Napoles P, Reina E, Turian J, Smith B and Bartholomew A: Mesenchymal stem cells enhance xenochimerism in NK-depleted hosts. Surgery 140: 315-321, 2006. 